\title{
Self in Time: Imagined Self-Location Influences Neural Activity Related to Mental Time Travel
}

\author{
Shahar Arzy, ${ }^{1,2,3}$ Istvan Molnar-Szakacs, ${ }^{1,4}$ and Olaf Blanke ${ }^{1,2}$ \\ ${ }^{1}$ Laboratory of Cognitive Neuroscience, Brain Mind Institute, Ecole Polytechnique Fédérale de Lausanne (EPFL), 1015 Lausanne, Switzerland, ${ }^{2}$ Department \\ of Neurology, University Hospital, 1211 Geneva, Switzerland, ${ }^{3}$ Department of Neurology, Hadassah Hebrew University Hospital, 91120 Jerusalem, Israel, \\ and ${ }^{4}$ Tennenbaum Center for the Biology of Creativity, Semel Institute for Neuroscience and Human Behavior, University of California, Los Angeles, \\ California 90095
}

Conscious awareness of the self as continuous through time is attributed to the human ability to remember the past and to predict the future, a cogitation that has been called "mental time travel" (MTT). MTT allows one to re-experience one's own past by subjectively "locating" the self to a previously experienced place and time, or to pre-experience an event by locating the self into the future. Here, we used a novel behavioral paradigm in combination with evoked potential mapping and electrical neuroimaging, revealing that MTT is composed of two different cognitive processes: absolute MTT, which is the location of the self to different points in time (past, present, or future), and relative MTT, which is the location of one's self with respect to the experienced event (relative past and relative future). These processes recruit a network of brain areas in distinct time periods including the occipitotemporal, temporoparietal, and anteromedial temporal cortices. Our findings suggest that in addition to autobiographical memory processes, the cognitive mechanisms of MTT also involve mental imagery and self-location, and that relative MTT, but not absolute MTT, is more strongly directed to future prediction than to past recollection.

Key words: mental time travel; autobiographical memory; future; occipitotemporal cortex; temporoparietal junction; spatial cognition

\section{Introduction}

A fundamental characteristic of human conscious experience is the ability to not only experience the present moment but also to recall the past and predict the future, or to "travel" back and forth in time, a facility that is called "mental time travel" (MTT) (Tulving, 1985, 2002; Atance and O'Neill, 2001; Levine, 2004; Levine et al., 2004). MTT to the past is proposed to rely on episodic autobiographical memory, allowing one to re-experience one's personal past through the subjective "location" of the self to a specific time and place that has previously been experienced (Tulving, 1985, 2002; Levine, 2004; Levine et al., 2004). Comparatively, MTT to the future is thought to be based on "episodic future thinking," or the ability to mentally locate the self forward to pre-experience an event (Ingvar, 1985; Atance and O'Neill, 2001; Okuda et al., 2003; Fellows and Farah, 2005). Converging evidence from recent memory research suggests that reexperiencing and pre-experiencing an event rely on similar neural mechanisms. Similar strategies and the same brain regions were found to be used in imagining past and future events, as

Received Dec. 24, 2007; revised May 15, 2008; accepted May 19, 2008.

This work was supported by the Fondation de Famille Sandoz (0.B.) and the Yeshaya Horowitz Association through The Center for Complexity Science (S.A.). The Cartool software (http://brainmapping.unige.ch/Cartool.htm) was programmed by Denis Brunet, Functional Brain Mapping Laboratory, The Center for Biomedical Imaging of Geneva and Lausanne, Geneva, Switzerland. We thank Dr. Yadin Dudai and Dr. Armin Schnider for helpful comments on previous versions of this manuscript, and Manuel Mercier for his help with data analysis.

Correspondence should be addressed to Dr. Shahar Arzy, Laboratory of Cognitive Neuroscience, Brain-Mind Institute, Ecole Polytechnique Fédérale de Lausanne, 1015 Lausanne, Switzerland. E-mail: shahar.arzy@epfl.ch.

DOI:10.1523/JNEUROSCI.5712-07.2008

Copyright $\odot 2008$ Society for Neuroscience $\quad$ 0270-6474/08/286502-06\$15.00/0 future predictions may be based on past memories (Atance and O’Neill, 2001; Okuda et al., 2003; Rugg et al., 2003; Fellows and Farah, 2005; Addis et al., 2007; Hassabis et al., 2007; Schacter and Addis, 2007; Szpunar et al., 2007).

However, when changing the location of one's self in time to past or future, one does not only recall and predict, but one also changes one's mental egocentric perspective on life events. Moreover, from these new self-locations in time, other life events might be regarded differently with respect to their relations to past or future. Thus, when imagining oneself as 10 years younger, last year's events are in the future (relative future) in relation to the initially imagined self-location in time, and vice versa (relative past). Therefore, to investigate MTT, we developed a novel behavioral paradigm by combining an autobiographical memory paradigm (Atance and O'Neill, 2001; Okuda et al., 2003; Addis et al., 2007; Szpunar et al., 2007) with an approach from experimental psychology on self-location and egocentric perspective taking (Ratcliff, 1979; Parsons, 1987; Zacks et al., 1999; Blanke et al., 2005; Arzy et al., 2006; Mohr et al., 2006). These latter studies showed behavioral and electrophysiological differences between judgments about one's own body while taken from one's actual spatial self-location versus different imagined self-locations. Considering lines of evidence that have already suggested shared mechanisms for processing time and space (Husserl, 1991; Coull and Nobre, 1998; Nobre, 2001; Merleau-Ponty, 2002; Buckner and Carroll, 2007), we hypothesized that self-location in time and space may also share cognitive and neural mechanisms. More specifically, we hypothesized that differences will be found not 


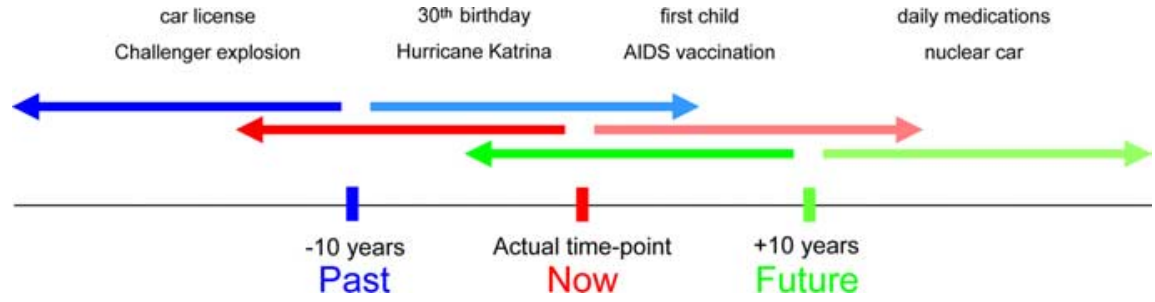

Figure 1. Stimuli and procedure. The three different self-locations in time (past, now, and future) are shown. Participants were asked to mentally imagine themselves in one of these self-locations, and from these self-locations to judge whether different self or nonself events (e.g., top row) already happened (relative past, darker colors) or are yet to happen (relative future, lighter colors).

only between different self-locations in time (past, now, and future) (Vogeley and Kupke, 2007), but also while imagining events in the relative past or the relative future. Here, we investigate these hypotheses and the neural correlates of MTT using behavioral measures, evoked potential (EP) mapping, and electrical neuroimaging in healthy adult participants.

\section{Materials and Methods}

Participants. Fourteen healthy volunteers (seven males, aged 29-38 years; mean \pm SD, $31.5 \pm 2.9$ years) participated in the experiment. All participants were right handed, and had normal or corrected-to-normal vision and no history of neurological or psychiatric disorders. All participants gave written informed consent before inclusion in the study, which was approved by the Ethical Committee of the University Hospital of Geneva (Switzerland).

Stimuli and procedures. The experimental manipulation asked participants to imagine themselves at three different self-locations in time: now (the present time), past (10 years in the past), or future (10 years in the future). In separate blocks for the past, now, and future self-locations, two-word phrases (average length of $13.6 \pm 2.5$ letters, mean \pm SD) describing different common events from personal life (e.g., car license; first child) or nonpersonal world events (e.g., Challenger explosion; hurricane Katrina) known to the participants were presented on a computer screen (Fig. 1). Note, that nonpersonal events are also intimately related to autobiographical memory for personal temporal, spatial, and mental information associated with these events; the terms "personal" and "nonpersonal" are used here in relative terms. Participants were asked to indicate whether the presented event took place before (relative past) or after (relative future) the currently imagined self-location in time (Fig. 1). For example, in the now or future blocks, participant should indicate "past" for the hurricane Katrina, but in the past block they should indicate "future," because it has not been 10 years since the event. However, if they are anticipating their first child in the next few years they should indicate "future" for "first child" in the past and now blocks but "past" in the future block. Thus, we examined MTT independently from the subject's self-location in time (past, now, or future) as the same event could be relatively in the past or in the future with respect to the imagined temporal self-location. Accordingly, we labeled this latter distinction relative MTT (rMTT), whereas the MTT between different self-locations in time was labeled absolute MTT (aMTT).

Stimuli were designed to be in the range of \pm 15 years of the imagined self-location in time. They included events that were chosen from most common personal life events for the personal items, and from major headline news events for the nonpersonal items, and appeared for $700 \mathrm{~ms}$ in the center of the computer screen with an interstimulus interval of $2000 \mathrm{~ms}$. Although previous behavioral, EP, and neuroimaging studies on MTT and autobiographical memory (Conway et al., 2001, 2003; Okuda et al., 2003; Rugg et al., 2003; Addis et al., 2007; Szpunar et al., 2007) presented stimuli for several seconds, to investigate self-location in time, presented stimuli here for relatively short epochs, similarly to previous experiments on self-location in space (Blanke et al., 2005; Arzy et al., 2006, 2007). Judgments were given using index and middle fingers of the left and right hand in alternating blocks as a button press on a serial response box. Participants were instructed to respond as quickly and precisely as possible while maintaining a mental image of themselves in the appropriate temporal self-location (past, now, or future), which were performed in six blocks (each repeated once) and counterbalanced across subjects. Each block included 120 stimuli, equally distributed among four groups: self (personal events) in relative past, self in relative future, nonself (world events) in relative past, and nonself in relative future, appearing in random order.

Analysis of behavioral data. Subsequent to the experiment, participants were asked to approximate the dates of the presented events. Correct responses were determined according to the estimated dates, as provided separately by each participant. Repeatedmeasures ANOVAs were run between participants on reaction time (RT) and error rate (ER) with the factors aMTT (past, now, and future), rMTT (relative past and relative future), and self (self and nonself events).

EEG recording, EP mapping, and distributed linear inverse solution. Continuous electroencephalography (EEG) recordings were acquired with a BioSemi system from 192 scalp electrodes (impedances, $<5 \mathrm{k} \Omega$ vertex referenced; $2048 \mathrm{~Hz}$ digitization; bandpass filtered, $0.1-100 \mathrm{H} \mathrm{z}$ ) in a darkened, electrically shielded booth. Epochs of EEG (from 0 to $800 \mathrm{~ms}$ after stimulus onset) were averaged for each of the experimental conditions in each subject to calculate the EPs. Trials with blinks or eye movements were rejected off-line, using horizontal and vertical electrooculograms. An artifact criterion of $\pm 100 \mu \mathrm{V}$ was applied at all other electrodes, and each EEG epoch was also visually evaluated before its inclusion in the EP. Before group averaging, EPs were bandpass filtered $(1-40 \mathrm{~Hz})$ and recalculated against the average reference. EP analysis was based on the examination of the spatial variations of the scalp voltage distribution over time and between conditions, an approach known as EP mapping (Michel et al., 2001, 2004; Blanke et al., 2005; Arzy et al., 2006). This approach searches for time segments of stable map topography that represent functional microstates of the brain during information processing. EP segments were defined by using a spatial $k$-means cluster analysis to identify the dominant map topographies on the scalp in the group-averaged EPs across the experimental conditions over time (Pascual-Marqui et al., 1995; Michel et al., 2001, 2004; Blanke et al., 2005; Arzy et al., 2006). These maps are the mean maps over the period where the segment was found. The optimal number of these template maps was determined by a modified cross-validation criterion (Pascual-Marqui et al., 1995). In a second step, the presence of a given EP map as identified in the group-averaged data were verified statistically in the EPs of the individual participants. This allowed us to determine the duration of a given EP map for each self-location in time across participants. These duration values for a given EP map can then be subjected to statistical analysis. Statistical comparisons were performed on the duration of each map (dependent variable) in the individual EPs using repeated-measures ANOVAs, with the factors aMTT, rMTT, and self. The same analysis was performed for the amplitude of the mean global field power of each map, which is defined as the spatial root mean squared across all electrodes (Lehmann and Skrandies, 1980). Finally, the neural generators for a given mean EP map were estimated by applying a distributed linear inverse solution, based on a local autoregressive average (LAURA model) (Grave de Peralta Menendez et al., 2004).

\section{Results}

\section{Behavior}

Data showed that RTs and ERs in past and future self-locations were significantly higher than in the now $\left(F_{(2,26)}=12.5, p<\right.$ $0.001 ; F_{(2,26)}=7.2, p<0.01$, respectively) (Fig. $2 A$ ). We refer to this effect for the three different self-locations in time as the aMTT effect. Interestingly, there was also a behavioral rMTT effect, where participants were always faster for relative future than for relative past events, with respect to their imagined temporal self-location, across all self-locations $\left(\mathrm{RTs}, F_{(1,13)}=18.9\right.$, 
$p<0.001$; ERs, $\left.F_{(1,13)}=5.8, p<0.01\right)$ (Fig. $2 A)$. There was no interaction of aMTT $X$ rMTT $\left(\mathrm{RTs}, F_{(2,26)}=0.34, p=0.71\right.$; ERs, $F_{(2,26)}=1.8, p=0.18$ ) (for more statistics and post hoc tests, see supplemental Tables 1,2 , available at www.jneurosci.org as supplemental material). Behavioral results also showed an effect of self (self-related faster than non-self-related events; $F_{(1,13)}$ $=28.64, p<0.001$ ) (supplemental Tables 1,2 , available at www.jneurosci.org as supplemental material). These effects are independent of one another as no interactions were found among them.

The aMTT effect corroborates previous behavioral data showing similar effects of MTT to past and future when asking subjects to imagine past and future events (Atance and O'Neill, 2001; Okuda et al., 2003; Addis et al., 2007; Szpunar et al., 2007). We provide novel evidence for response facilitation for relative future events (rMTT effect), which are faster and more accurate. Note that this was found independent of the actual events imagined. This suggests that mental processing of events is future oriented across all investigated self-locations in time as has recently been speculated (Dudai and Carruthers, 2005; Schacter and Addis, 2007).

\section{Electrical neuroimaging}

EP mapping of the group-averaged data revealed a distinct brain segment of stable voltage topography (or EP map) that distinguished the past and future from the now self-location. The map appeared between 500 to $600 \mathrm{~ms}$ after stimulus presentation (Fig. 2 B, segment 14) and lasted significantly longer in the past and future selflocations than in the now (aMTT effect; $F_{(2,26)}=3.8 ; p=0.03$ ) (Fig. $2 B, C$ ). In further agreement with response facilitation for relative future events, this brain activation was shorter for relative future than relative past events (rMTT effect; $F_{(1,13)}=$ $5.6 ; p=0.01$ ) independently of the imagined self-location in time (no significant interaction, $F_{(2,26)}=0.9 ; p=0.42$ ) (Fig. $2 C$, right) (supplemental table 3 , available at www.jneurosci.org as supplemental material). A self effect (shorter brain activity for self-related than non-self-related events) was also found for this $\operatorname{EP} \operatorname{map}\left(F_{(1,13)}=4.7 ; p=0.02\right)$ with no significant interaction $\left(F_{(2,26)}=1.0 ; p=0.32\right)$ (supplemental Table 3, available at www.jneurosci.org as supplemental material), in-line with the behavioral data. There were no significant modulations with respect to the strength of brain activations in the different self-locations (supplemental Table 3, available at www.jneurosci.org as supplemental material). A linear inverse solution (Grave de Peralta Menendez et al., 2004) localized this map to regions of the left anteromedial temporal cortex, right temporoparietal junction, and occipitotemporal cortex
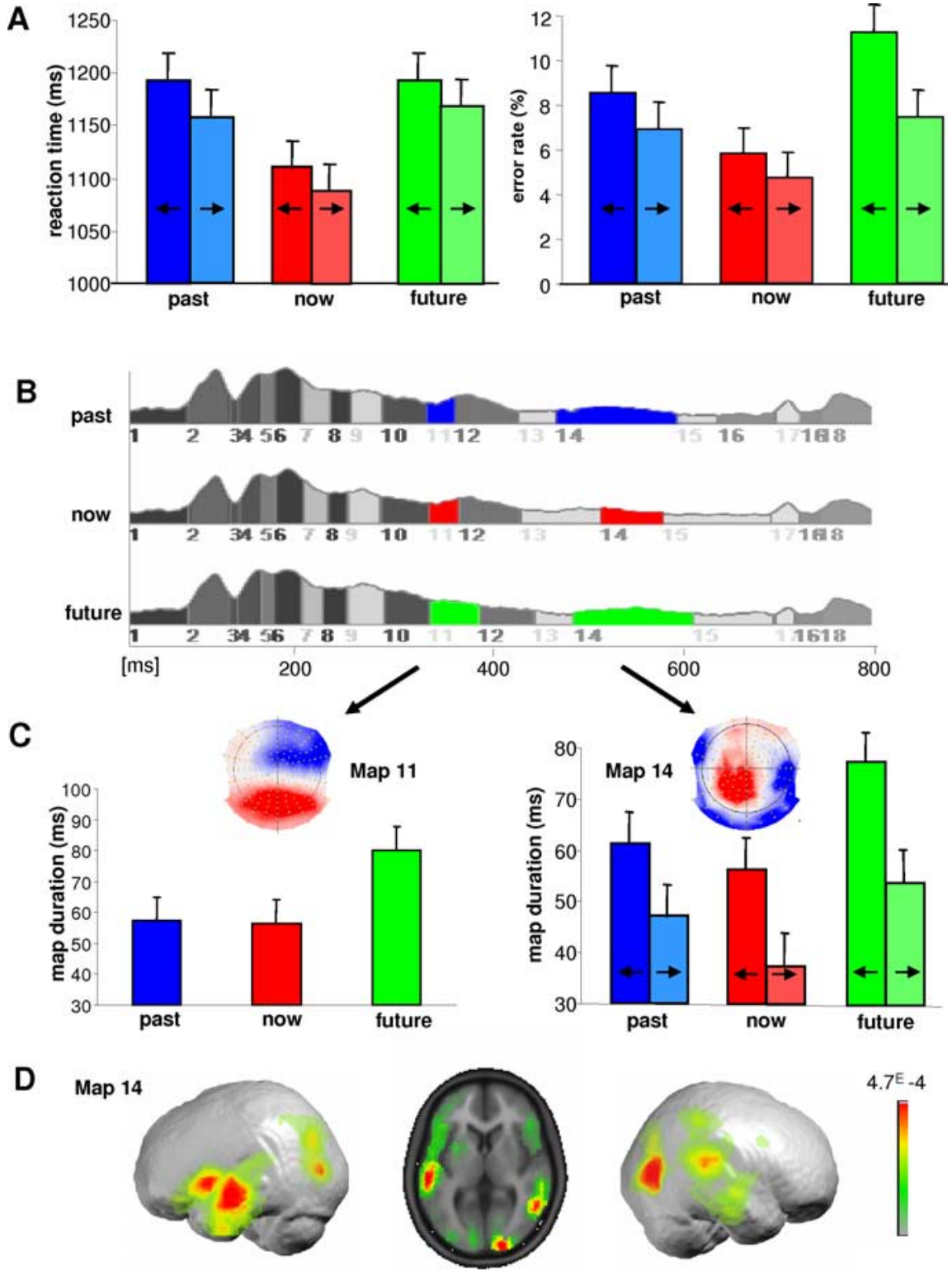

Figure 2. Behavioral and EP data. A, RTs and ERs are plotted separately for past (blue), now (red), and future (green) selflocations in time. Error bars indicate intersubject SEM. Note the significantly higher RTs and ERs for past and future with respect to the now. In addition, in all self-locations (past, now, future) RTs and ERs were higher for the relative past (left bars, darker colors) than the relative future (right bars, lighter colors). $\boldsymbol{B}$, Segments of stable map topography in the three temporal selflocations under the global field power curve from 0 to $800 \mathrm{~ms}$. EP segment 11 (future map) was found at $\sim 300-400 \mathrm{~ms}$ and was significantly longer for future self-location (green) than for the past (blue) and now (red). EP segment 14 (MTT map) was found at $\sim 500-600 \mathrm{~ms}$ and was significantly longer for past and future self-locations than for the now. C, Map topography and duration of MTT map (right) and future map (left) for the three experimental self-locations for all participants. Note that duration of the MTT map parallels the behavioral results. In all temporal self-locations MTT map duration was longer for the relative past (left bars, darker colors) than for the relative future (right bars, lighter colors). $\boldsymbol{D}$, Generators of MTT map were localized to the right temporoparietal, occipitotemporal, and left anteromedial temporal cortices.

(Fig. 2D). Notably, only this EP map showed effects of aMTT, rMTT, and self, reflecting behavioral data, suggesting that this map most closely reflects neural activity associated with mental time travel (MTT map).

An earlier map appearing between 300 to $400 \mathrm{~ms}$ after stimulus onset (Fig. 2 B, segment 11 ) was significantly longer and stronger in the future than in the past and now self-locations (future effect; duration, $F_{(2,26)}=4.2, p=0.02$ ) (Fig. $2 C$, left). The duration and strength of this brain activation did not show a significant effect of rMTT (duration, $F_{(1,13)}=0.8, p=0.4$ ) and no signif- 
icant interaction (duration, $F_{(1,13)}=0.9, p=0.4$ ) or self effect (duration, $F_{(1,13)}=0.4, p=0.7$ ) (for strength statistics, see supplemental Table 4, available at www.jneurosci.org as supplemental material). The same linear inverse solution localized this segment to the occipitotemporal cortex bilaterally (supplemental Fig. 1, available at www.jneurosci.org as supplemental material). This brain activity precedes the MTT map, and is significantly associated with the future self-location, but not other selflocations or an effect of self.

To summarize, these EP data corroborate the behavioral results, with an EP map at 500-600 ms after stimulus onset showing independent effects of aMTT (different self-locations in time: past, now, and future), rMTT (relative future or relative past with respect to the imagined self-location in time), and self (selfrelated vs non-self-related events). In addition, an earlier EP map, at 300-400 ms after stimulus onset, was found to be associated only with the future self-location in time. These MTT associated cognitive processes recruit occipitotemporal, temporoparietal, and anteromedial temporal cortices between 300 and $600 \mathrm{~ms}$ after stimulus onset.

\section{Discussion}

In this study, we designed a paradigm combining concepts from autobiographical memory research, mental imagery, and selflocation to investigate MTT to the past and future. This paradigm allowed us to disentangle mechanisms of MTT and self-location in time, at least partly, from memory related mechanisms, in behavioral and neural terms. The paradigm asks participants to change their self-location in time to the past, the now, or the future (aMTT), and then to determine whether events happened before (relative past) or will happen after (relative future) the imagined self-location in time (rMTT). These two aspects of MTT were reflected at the behavioral level, as RTs and ERs were higher for past and future self-locations in time than in the now self-location (aMTT effect). In addition, in all self-locations in time, participants were faster and more accurate to the relative future than relative past (rMTT effect). These effects were also borne out at the neural level (MTT map), as at $\sim 500 \mathrm{~ms}$ after stimulus presentation a network consisting of the anteromedial temporal, temporoparietal, and occipitotemporal cortices was activated in the past and future self-locations longer than in the now (aMTT), and in all self-locations this activation was shorter for relative future events than relative past events (rMTT).

The aMTT effect in the current study confirms that MTT to the past and future share brain mechanisms and is in line with previous work (Atance and O'Neill, 2001; Okuda et al., 2003; Addis et al., 2007; Buckner and Carroll, 2007; Hassabis et al., 2007; Szpunar et al., 2007). The novel rMTT effect, in which participants were always faster and more accurate for relative future than relative past events, independent of their temporal self-location, provides experimental evidence for proposals that MTT is used principally to anticipate future occurrences (Schacter, 2001; Schacter et al., 2003; Dudai and Carruthers, 2005; Schacter and Addis, 2007). This is also supported by the activation of the occipitotemporal cortex that was primarily found for future self-location (future map), peaking as early as 300-400 ms after stimulus presentation, therefore indicating early processing for future events. These activations were found earlier than those reported in previous EP studies on autobiographical memory (Conway et al., 2001, 2003; Rugg et al., 2003). Although longer processing times might be important for the full construction of autobiographical memory and MTT as assessed in these previous studies, the present approach allows to study
MTT and self-location in time during shorter time-periods. We speculate that both approaches may rely on the activation of partly different brain networks, but would argue that the crucial or sufficient brain areas encoding MTT involve self-location in time, and are therefore engaged when using the present experimental strategy. The importance of short time periods in MTT is also suggested by the observation that MTT is crucial for quick decision making (Boyer, 2008), as is simulated in the current study.

Our data also showed a self effect in which participants responded significantly faster to self-related than non-self-related events, an effect that we also found in our EP data as we found shorter brain activity at the anteromedial temporal, temporoparietal, and occipitotemporal cortices for self-related than nonself-related events. Indeed, psychological data have suggested that self-representation involves personal identity and continuity across time. The "narrative" self, which is a self-representation constituted with past and future in stories that we and others tell about ourselves (Dennett, 1991; Gallagher, 2000), and the "extended" self, based on memory and anticipation (Neisser, 1995), may be related concepts. As cognitive representations of the self develop across time, information from past experiences becomes incorporated in self-representation (Markus, 1977). Access to this information is privileged, with subjects showing twice as efficient recall of self-related items than non-self-related items in a phenomenon labeled the self reference effect (Symons and Johnson, 1997), which may also be relevant to our findings. However, the future map, which did not show main effects or interactions involving self/nonself distinction, suggests that there are perspectives from which there may be less distinction between self- and non-self-related events.

Our EP recordings show that self-location in time recruits a distributed neural network including the occipitotemporal, anteromedial temporal, and temporoparietal cortices. The activation of the occipitotemporal cortex is in agreement with previous neuroimaging studies that found recruitment of the occipitotemporal cortex in autobiographical memory tasks (Maguire, 2001; Conway et al., 2002; Svoboda et al., 2006; Addis et al., 2007). Some have proposed that the occipitotemporal cortex might be involved in recall through visual imagery (Rubin and Greenberg, 1998; Conway et al., 2002; Slotnick and Schacter, 2006; Svoboda et al., 2006). In addition, clinical studies have described amnesia with isolated or predominant damage to striate and occipitotemporal cortex (Ogden, 1993; Hunkin et al., 1995). Together, these data suggest that occipitotemporal cortex plays a central role in MTT and self-location in time.

Activation was also found in anteromedial temporal cortex, which has been proposed to be a crucial structure for processing both semantic knowledge and autobiographical information, as found in clinical and neuroimaging studies (Corkin, 2002; Levine et al., 2004; Squire et al., 2004; Svoboda et al., 2006). Our data suggest that this area may also play a key role in MTT, which is based on similar cognitive processes (Okuda et al., 2003; Addis et al., 2007; Buckner and Carroll, 2007), as was shown recently also in amnestic patients (Hassabis et al., 2007).

Finally, we show that the temporoparietal junction is activated during temporal judgments from different self-locations in time. This region has been shown previously to be involved in many self-related tasks such as visuospatial perspective taking (Ruby and Decety, 2001; Vogeley and Fink, 2003), mental own-body imagery (Zacks et al., 1999), and own-action recognition (Farrer and Frith, 2002). Particularly, previous work has shown that the temporoparietal junction is a structure crucial for self-location in 
space, as participants showed different behavioral patterns and brain activities while imagining themselves from their own bodily spatial self-location or from an external bodily self-location, as well as while imagining looking at themselves from the front or back (Zacks et al., 1999; Ruby and Decety, 2001; Vogeley and Fink, 2003; Blanke et al., 2005; Arzy et al., 2006, 2007). The current data extend these findings by showing that the temporoparietal junction encodes not only self-location in space but also self-location in time. Finally, in addition to the similarity in brain activity between spatial and temporal self-processing, the time window of electrophysiological correlates of aMTT and rMTT overlaps with those described in previous event-related studies on self-location and mental imagery of self in space (Zacks et al., 1999; Coull et al., 2000; Nobre, 2001; Blanke et al., 2005; Arzy et al., 2006, 2007). This spatiotemporal electrophysiological similarity also supports the hypothesis that MTT may not only involve memory mechanisms, but also mechanisms related to mental imagery and self-location.

In conclusion, in the present study we found two independent components of MTT: absolute MTT, which is the mentally imagined self-location in time (past, now, and future), and relative MTT, which is the relation between this temporal self-location and the experienced event (relative past or relative future). The latter effect suggests that mental processing of events is more strongly directed to future prediction than to past recollection, as participants were faster and more accurate for relative future than relative past events across all self-locations in time. Moreover, of all tested temporal self-locations, future was also processed earliest at the neural level. This may indicate that the neural systems subserving MTT have evolved to anticipate and pilot our behavior rather than primarily encoding the past (Haselton and Nettle, 2006; Boyer, 2008). Finally, MTT was found to be processed faster for self-related than non-self-related events, although both were processed with respect to the subject's imagined selflocation in time. Three brain regions were found to be recruited for self-location in time: the occipitotemporal cortex, contributing to MTT, mental imagery, and visual memory, the temporoparietal junction, which is a crucial region for self-related processing and self-location, and the anteromedial temporal cortex, a core region in memory and spatial processing. This network of regions indicates how MTT requires not only memory skills but also self-location and mental imagery elements.

\section{References}

Addis DR, Wong AT, Schacter DL (2007) Remembering the past and imagining the future: common and distinct neural substrates during event construction and elaboration. Neuropsychologia 45:1363-1377.

Arzy S, Thut G, Mohr C, Michel CM, Blanke O (2006) Neural basis of embodiment: distinct contributions of temporoparietal junction and extrastriate body area. J Neurosci 26:8074-8081.

Arzy S, Mohr C, Michel CM, Blanke O (2007) Duration and not strength of activation in temporo-parietal cortex positively correlates with schizotypy. NeuroImage 35:326-333.

Atance CM, O’Neill DK (2001) Episodic future thinking. Trends Cogn Sci 5:533-539.

Blanke O, Mohr C, Michel CM, Pascual-Leone A, Brugger P, Seeck M, Landis $\mathrm{T}$, Thut G (2005) Linking out-of-body experience and self processing to mental own-body imagery at the temporoparietal junction. J Neurosci 25:550-557.

Boyer P (2008) Evolutionary economics of mental time travel? Trends Cogn Sci, in press.

Buckner RL, Carroll DC (2007) Self-projection and the brain. Trends Cogn Sci 11:49-57.

Conway MA, Pleydell-Pearce CW, Whitecross SE (2001) The neuroanatomy of autobiographical memory: a slow cortical potential study of autobiographical memory retrieval. J Mem Lang 45:493-524.
Conway MA, Pleydell-Pearce CW, Whitecross S, Sharpe H (2002) Brain imaging autobiographical memory. Psychol Learn Motiv 41:229-264.

Conway MA, Pleydell-Pearce CW, Whitecross SE, Sharpe H (2003) Neurophysiological correlates of memory for experienced and imagined events. Neuropsychologia 41:334-340.

Corkin S (2002) What's new with the amnesic patient H.M.? Nat Rev Neurosci 3:153-160.

Coull JT, Nobre AC (1998) Where and when to pay attention: the neural systems for directing attention to spatial locations and to time intervals as revealed by both PET and fMRI. J Neurosci 18:7426-7435.

Coull JT, Frith CD, Buchel C, Nobre AC (2000) Orienting attention in time: behavioural and neuroanatomical distinction between exogenous and endogenous shifts. Neuropsychologia 38:808-819.

Dennett D (1991) Consciousness explained. Boston: Little Brown.

Dudai Y, Carruthers M (2005) The Janus face of Mnemosyne. Nature 434:567.

Farrer C, Frith CD (2002) Experiencing oneself vs another person as being the cause of an action: the neural correlates of the experience of agency. NeuroImage 15:596-603.

Fellows LK, Farah MJ (2005) Dissociable elements of human foresight: a role for the ventromedial frontal lobes in framing the future, but not in discounting future rewards. Neuropsychologia 43:1214-1221.

Gallagher II (2000) Philosophical conceptions of the self: implications for cognitive science. Trends Cogn Sci 4:14-21.

Grave de Peralta Menendez R, Murray MM, Michel CM, Martuzzi R, Gonzalez Andino SL (2004) Electrical neuroimaging based on biophysical constraints. NeuroImage 21:527-539.

Haselton MG, Nettle D (2006) The paranoid optimist: an integrative evolutionary model of cognitive biases. Pers Soc Psychol Rev 10:47-66.

Hassabis D, Kumaran D, Vann SD, Maguire EA (2007) Patients with hippocampal amnesia cannot imagine new experiences. Proc Natl Acad Sci USA 104:1726-1731.

Hunkin NM, Parkin AJ, Bradley VA, Burrows EH, Aldrich FK, Jansari A, Burdon-Cooper C (1995) Focal retrograde amnesia following closed head injury: a case study and theoretical account. Neuropsychologia 33:509-523.

Husserl E (1991) The lectures on the consciousness of internal time from the year 1905. In: On the phenomenology of the consciousness of internal time (1893-1917), pp 3-75. London/Dordrecht: Kluwer.

Ingvar DH (1985) "Memory of the future": an essay on the temporal organization of conscious awareness. Hum Neurobiol 4:127-136.

Lehmann D, Skrandies W (1980) Reference-free identification of components of checkerboard-evoked multichannel potential fields. Electroencephalogr Clin Neurophysiol 48:609-621.

Levine B (2004) Autobiographical memory and the self in time: brain lesion effects, functional neuroanatomy, and lifespan development. Brain Cogn 55:54-68.

Levine B, Turner GR, Tisserand D, Hevenor SJ, Graham SJ, McIntosh AR (2004) The functional neuroanatomy of episodic and semantic autobiographical remembering: a prospective functional MRI study. J Cogn Neurosci 16:1633-1646.

Maguire EA (2001) Neuroimaging studies of autobiographical event memory. Philos Trans R Soc Lond B Biol Sci 356:1441-1451.

Markus H (1977) Self-schemata and processing information about the self. J Pers Soc Psychol 35:63-78.

Merleau-Ponty M (2002) Phenomenology of perception. London: Routledge.

Michel CM, Thut G, Morand S, Khateb A, Pegna AJ, Grave de Peralta R, Gonzalez S, Seeck M, Landis T (2001) Electric source imaging of human brain functions. Brain Res Brain Res Rev 36:108-118.

Michel CM, Murray MM, Lantz G, Gonzalez S, Spinelli L, Grave de Peralta R (2004) EEG source imaging. Clin Neurophysiol 115:2195-2222.

Mohr C, Blanke O, Brugger P (2006) Perceptual aberrations impair mental own-body transformations. Behav Neurosci 120:528-534.

Neisser U (1995) Criteria for an ecological self. In: The self in infancy: theory and research (Rochat P, ed), pp 17-30. Amsterdam: Elsevier.

Nobre AC (2001) Orienting attention to instants in time. Neuropsychologia 39:1317-1328.

Ogden JA (1993) Visual object agnosia, prosopagnosia, achromatopsia, loss of visual imagery, and autobiographical amnesia following recovery from cortical blindness: case M.H. Neuropsychologia 31:571-589.

Okuda J, Fujii T, Ohtake H, Tsukiura T, Tanji K, Suzuki K, Kawashima R, 
Fukuda H, Itoh M, Yamadori A (2003) Thinking of the future and past: the roles of the frontal pole and the medial temporal lobes. NeuroImage 19:1369-1380.

Parsons LM (1987) Imagined spatial transformation of one's body. J Exp Psychol Gen 116:172-191.

Pascual-Marqui RD, Michel CM, Lehmann D (1995) Segmentation of brain electrical activity into microstates: model estimation and validation. IEEE Trans Biomed Eng 42:658-665.

Ratcliff G (1979) Spatial thought, mental rotation and the right cerebral hemisphere. Neuropsychologia 17:49-54.

Rubin DC, Greenberg DL (1998) Visual memory-deficit amnesia: a distinct amnesic presentation and etiology. Proc Natl Acad Sci USA 95:5413-5416.

Ruby P, Decety J (2001) Effect of subjective perspective taking during simulation of action: a PET investigation of agency. Nat Neurosci 4:546-550.

Rugg MD, Herron JE, Morcom AM (2003) Electrophysiological studies of retrieval processing. In: Neuropsychology of memory (Squire LR, Schacter DL, eds), pp 154-165. New York: Guilford.

Schacter DL (2001) Seven sins of memory: how the mind forgets and remembers. New York: Houghton Mifflin.

Schacter DL, Addis DR (2007) Constructive memory: the ghosts of past and future. Nature 445:27.

Schacter DL, Chiao JY, Mitchell JP (2003) The seven sins of memory: implications for self. Ann NY Acad Sci 1001:226-239.
Slotnick SD, Schacter DL (2006) The nature of memory related activity in early visual areas. Neuropsychologia 44:2874-2886.

Squire LR, Stark CE, Clark RE (2004) The medial temporal lobe. Annu Rev Neurosci 27:279-306.

Svoboda E, McKinnon MC, Levine B (2006) The functional neuroanatomy of autobiographical memory: a meta-analysis. Neuropsychologia 44:2189-2208

Symons CS, Johnson BT (1997) The self-reference effect in memory: a meta-analysis. Psychol Bull 121:371-394.

Szpunar KK, Watson JM, McDermott KB (2007) Neural substrates of envisioning the future. Proc Natl Acad Sci USA 104:642-647.

Tulving E (1985) Memory and consciousness. Can Psychol 26:1-12.

Tulving E (2002) Episodic memory: from mind to brain. Annu Rev Psychol 53:1-25.

Vogeley K, Fink GR (2003) Neural correlates of the first-personperspective. Trends Cogn Sci 7:38-42.

Vogeley K, Kupke C (2007) Disturbances of time consciousness from a phenomenological and a neuroscientific perspective. Schizophr Bull 33:157-165.

Zacks J, Rypma B, Gabrieli JD, Tversky B, Glover GH (1999) Imagined transformations of bodies: an fMRI investigation. Neuropsychologia 37: $1029-1040$. 